\title{
Acoustic reference library of mexican insectivorous bats: Phase I.
}

El 15 de marzo de 2016 se firmó el Convenio Número FB1796/ME004/16 entre la Comisión Nacional para el Conocimiento y Uso de la Biodiversidad (CONABIO) y la Asociación Mexicana de Mastozoología A. C. (AMMAC), para ejecutar el proyecto "Compilación de fonoteca de referencia de los murciélagos insectívoros de México: Fase I". El objetivo principal del proyecto fue iniciar la conformación de una fonoteca de referencia de sonidos de ecolocalización de las especies de murciélagos con sonidos de alta intensidad presentes en el territorio mexicano (SonozotzAMMAC-CONABIO 2018). A dos años de haberse iniciado, el 28 de febrero de 2018, el proyecto técnicamente ha finalizado con resultados exitosos y la obtención de los productos comprometidos.

Desde la reunión inicial en 2014 que se efectuó en el marco del XI Congreso Nacional de Mastozoología en la Benemérita Universidad Autónoma de Puebla, la organización del proyecto supuso un gran reto para conjuntar los esfuerzos que diversos especialistas y miembros de la AMMAC venían desarrollando en la detección acústica de murciélagos. El esfuerzo tenía que ser multiinstitucional, abarcar todo México, incluir innumerables tipos de ecosistemas, considerar más de 80 especies de murciélagos que emiten pulsos de ecolocalización intensos, conformar grupos de trabajo con investigadores con diferentes formas pensar, incluir dependencias con equipo y capacidades técnicas disímiles, así como establecer procedimientos de gestión estandarizados entre instancias con manuales de administración distintos. Todo tendría que ser planeado y ejecutado con muy elevados estándares de calidad técnica y científica.

Para la AMMAC, el proyecto representó también el primero en donde la Asociación fungió como institución receptora de los recursos financieros y como responsable de la ejecución de los mismos. El aprendizaje en la gestión y administración de los fondos, marcan un precedente positivo y abren oportunidades para poder someter futuros proyectos con cobertura nacional e impacto científico internacional. De esta forma, los resultados no sólo radican en productos académicos (informes, tesis, publicaciones, formación de recursos humanos, congresos, bases de datos, metadatos, fotografías, etc.), sino también en buenas prácticas estandarizadas para la gestión y la aplicación de los resultados (procedimientos, protocolos, etc.). Por ejemplo, se desarrollaron procedimientos por parte de la AMMAC para administrar recursos financieros provenien- tes de Proyectos de Investigación Científica (PIC), se actualizaron los estatutos de la Asociación, se mejoró el manejo contable y se generaron convenios de colaboración con algunas de las instituciones a las cuales están adscritos los miembros participantes.

Desde un inicio, el proyecto contó con la participación de por lo menos 18 miembros de la AMMAC pertenecientes a igual número de instituciones distribuidas en todo el territorio mexicano. Se abarcó ocho regiones del país: Californiana (Baja California, Baja California Sur y Sonora); Noroeste (Durango, Sinaloa y Chihuahua); Occidente (Colima, Nayarit y Jalisco); Oriente (Puebla, Tlaxcala y Veracruz): Centro Norte (Aguascalientes, Guanajuato, San Luis Potosí, Nuevo León y Zacatecas); Centro Sur (Estado de México, Morelos, Hidalgo y Querétaro); Sureste (Campeche, Quintana Roo y Yucatán) y Suroeste (Chiapas, Oaxaca y Tabasco). Fue necesario generar acuerdos para nombrar al responsable del proyecto, a los coordinadores generales técnicos y a los coordinadores para cada región. El proceso exigió la voluntad de todos los participantes para maximizar consensos y minimizar conflictos.

El proyecto planteo siete objetivos particulares (Sonozotz-AMMAC-CONABIO 2018), que de forma sintética incluían la conformación de una red de grupos de trabajo con personal capacitado; organizar talleres de capacitación y entrenamiento; integrar grabaciones acústicas de murciélagos ya existentes; definir un diseño de muestreo adecuado; diseñar un procedimiento de validación e ingreso de datos a la fonoteca; incorporar a la fonoteca las primeras grabaciones; y determinar los procedimientos de comparación estadística para la discriminación entre especies.

Los participantes en el proyecto denominaron a esta iniciativa "Sonozotz". "Sono" hace alusión a los sonidos de ecolocalización emitidos por los murciélagos; "Zotz", significa murciélago en lengua maya y hace referencia a los importantes valores iconográficos, religiosos, simbólicos y míticos de los quirópteros vinculados con la gran diversidad cultural de México; AMMAC, además del significado de las siglas, representa los esfuerzos colegiados e institucionales de los mastozoólogos del país. En el 2016, a través de un concurso abierto, durante el XIII Congreso Nacional de Mastozoología en la Universidad de Ciencias y Artes de Chiapas, "Sonozotz" adquirió logotipo.

Como parte del proyecto, se generó un protocolo general de trabajo de campo que permitió la homogenización de la colecta de información, el procesamiento y la organización 
de los datos de campo, así como formatos e instructivos para la descripción de las localidades, los datos de ejemplares, los datos de grabación y el catálogo de preparación. El protocolo incluyó una guía sobre el proceso de grabación de los sonidos, la utilización de los equipos, la instalación de los programas especializados, el ensamblado y activación de los detectores, los métodos de grabación y liberación de ejemplares, la colecta de tejidos y de ejemplares para su posterior resguardo en las colecciones científicas de mamíferos autorizadas. Este protocolo representa por sí mismo un logro del proyecto, que permitirá a otros grupos interesados en acústica aplicarlo en diversos estudios.

Se realizaron cuatro talleres en diferentes sedes: 1) La estación Biológica La Mancha del Instituto de Ecología, en La Mancha, Veracruz; 2) Centro Interdisciplinario de Investigación para el Desarrollo Integral Regional (CIIDIR) Unidad Durango del Instituto Politécnico Nacional (IPN), en Durango, Durango; CIIDIR-IPN Unidad Oaxaca, Oaxaca; Centro de Investigación en Biodiversidad y Conservación (CIByC) de la Universidad Autónoma del Estado de Morelos (UAEM), Cuernavaca, Morelos. En todos los talleres asistieron aproximadamente 80 personas que desarrollan trabajos de investigación con murciélagos en México y que participaron en sesiones prácticas para la captura, muestreo y grabación de ejemplares capturados in situ.

Durante las salidas de campo que se realizaron de junio de 2016 a diciembre de 2017, se aplicó el protocolo de muestreo específicamente elaborado para el proyecto. Los grupos participantes por región, tuvieron la oportunidad de contar con el apoyo de estudiantes y voluntarios. En ese sentido, tanto los talleres como el trabajo de campo y gabinete, tuvieron un importante efecto en la formación de recursos humanos de licenciatura y posgrado de diversas instituciones del país.

El proyecto logró reunir 173 colectores que participaron al menos en una salida de campo, se cubrió el $100 \%$ de las ocho regiones propuestas con 183 localidades que cubrieron los principales ecosistemas de México. Se colectaron 1,640 individuos pertenecientes a 67 especies y se cubrió el registro de llamados de ecolocalización del $63 \%$ de las especies de murciélagos insectívoros de México con un total de 2,302 grabaciones (Sonozotz-AMMAC-CONABIO 2018).

La CONABIO desarrolló una plataforma especialmente diseñada para este proyecto en donde estarán albergadas bases de datos, mapas, metadatos, llamados y fotografías, que en el corto plazo constituirá la primera fonoteca de referencia de los murciélagos insectívoros de México y la primera a nivel mundial de un país megadiverso. Se espera que este proyecto permita plantear un programa de monitoreo a nivel nacional y consolidar la formación de la "Red Mexicana de Monitoreo Acústico de Murciélagos".

La aplicación de las metodologías de detección acústica en países como México todavía está limitada por la poca disponibilidad de equipo y, sobre todo, porque no se con- taba con fonotecas de referencia adecuadas para la elaboración de criterios de identificación de las especies (Walters et al. 2013). En ese sentido, la compilación de una fonoteca de sonidos de ecolocalización representativa de las especies presentes en el país, así como el desarrollo de criterios de identificación acústica que pudieran estar disponibles como herramientas electrónicas de uso libre, potenciará el uso de los métodos de detección acústica.

Los murciélagos insectívoros son susceptibles a amenazas generadas por la actividad humana. Es evidente que estas especies son sensibles a la pérdida y degradación de los hábitats naturales, a los pesticidas y contaminantes (Racey y Entwistle 2003). Muchas especies son excepcionalmente sensibles a impactos con los generadores de energía eólica (Kunz et al. 2007) y otras son especialmente vulnerables a las perturbaciones de sus refugios (Racey $y$ Entwistle 2003).

La disponibilidad de una herramienta para la identificación acústica y de metodologías de monitoreo estandarizadas (Zamora-Gutiérrez et al. 2016), facilitará la operación y la obtención de datos confiables a las empresas, agencias gubernamentales, academia y otros sectores interesados en la evaluación de riesgos e impactos a través del estudio de los murciélagos. Esto permitirá la generación de información sobre la distribución de las especies y posibilitará la aplicación de programas de monitoreo y estudios técnicos requeridos para la implementación de proyectos de desarrollo, como Manifiestos de Impacto Ambiental (MIA), Documentos Técnicos Unificados (DTU), Estudios Técnicos Justificativos (ETJ), Auditorías Ambientales (AA), Programas de Desarrollo Urbano (PDU), Programas de Manejo de Áreas Naturales Protegidas y de Unidades de Manejo de Vida Silvestre, entre otros.

La AMMAC agradece el apoyo recibido por la CONABIO, así como el trabajo realizado por todos los participantes en el proyecto y a todas las instituciones a los que están adscritos los colaboradores. Además de los productos académicos, se espera que en el corto plazo, se planteen otros proyectos relativos a la conservación y manejo de los mamíferos de México, con la participación activa de los miembros de la AMMAC.

\section{Literatura citada}

Kunz, T. H., E. B. Arnett, y B. M. Cooper. 2007. Assessing Impacts of Wind-Energy Development on Nocturnally Active Birds and Bats: A Guidance Document. Journal of Wildlife Management 71:2449-2486.

Racey, P. A., y A. C. Entwistle. 2003. Conservation ecology of bats. Pp. 680-743 in Bat ecology (Kunz, T. H., y M. B. Fenton, eds.). University of Chicago Press. Chicago, EE.UU.

Sonozotz-AMMAC-CONABIO (Sonozotz-Asociación Mexicana de Mastozoología-Comisión Nacional para el Conocimiento y Uso de la Biodiversidad). 2018. Informe final del proyecto Compilación de fonoteca de referencia de murciélagos insectívoros de México: Fase I. Informe no publicado. AMMAC, CONABIO. Ciudad de México, México. 
Walters, C. L., A. Collen, T. Lucas, K. Mroz, C. A. Sayer, y K. E. Jones. 2013. Challenges of Using Bioacoustics to Globally Monitor Bats. Pp. 479-499 in Bat Evolution, Ecology, and Conservation (Adams, R. A., y S. C. Pedersen, eds.). Springer Science Business Media. New York, EE.UU.

Zamora-Gutiérrez, V., C. López-González, M. C. Mac Swiney G., B. M. Fenton, G. Jones, E. K. Kalko, S. J. Puechmaille, V. Stathopoulos, y K. E. JONES. 2016. Acoustic identification of Mexican bats based on taxonomic and ecological constraints on call design. Methods in Ecology and Evolution 7:1082-1091.

JaVier EnRIQue Sosa-Escalante

Asociación Mexicana de Mastozoología A. C. Hacienda Vista Hermosa 107, Colonia Villa Quietud, Delegación Coyoacán, CP. 04960. Ciudad de México, México. Email: ammac.presidencia@gmail.com (JESE) 
FONOTECA ACÚSTICA DE MURCIÉLAGOS

200 THERYA Vol. 9 (3): 197-200 